\title{
Equation of State of Ultracold Fermions in the 2D BEC-BCS Crossover Region
}

\author{
I. Boettcher, ${ }^{1, *}$ L. Bayha, ${ }^{2}$ D. Kedar, ${ }^{2}$ P. A. Murthy, ${ }^{2}$ M. Neidig, ${ }^{2}$ M. G. Ries, ${ }^{2}$ A. N. Wenz, ${ }^{2}$ \\ G. Zürn, ${ }^{2}$ S. Jochim, ${ }^{2}$ and T. Enss ${ }^{1}$ \\ ${ }^{1}$ Institute for Theoretical Physics, Heidelberg University, D-69120 Heidelberg, Germany \\ ${ }^{2}$ Physikalisches Institut, Heidelberg University, D-69120 Heidelberg, Germany
}

(Received 11 September 2015; published 27 January 2016)

\begin{abstract}
We report the experimental measurement of the equation of state of a two-dimensional Fermi gas with attractive $s$-wave interactions throughout the crossover from a weakly coupled Fermi gas to a Bose gas of tightly bound dimers as the interaction strength is varied. We demonstrate that interactions lead to a renormalization of the density of the Fermi gas by several orders of magnitude. We compare our data near the ground state and at finite temperature with predictions for both fermions and bosons from quantum Monte Carlo simulations and Luttinger-Ward theory. Our results serve as input for investigations of close-to-equilibrium dynamics and transport in the two-dimensional system.
\end{abstract}

DOI: 10.1103/PhysRevLett.116.045303

The rich phenomenology of fermionic many-body systems reveals itself on very different scales of energy, ranging from solid state materials and ultracold quantum gases to heavy-ion collisions and neutron stars. Understanding the underlying mechanisms promises substantial advances both on a fundamental and technological level. Ultracold quantum gases provide a platform for the exploration of the macroscopic phases and thermodynamic properties of fermionic many-body Hamiltonians in a highly controlled manner [1]. In particular, using strongly anisotropic traps, it is possible to enter the 2D regime [2-7] which is of large interest to the condensed matter community $[8,9]$.

The thermodynamic properties of a many-body system are encapsulated in its equation of state (EOS) $n\left(\mu, T,\left\{g_{i}\right\}\right)$, which expresses the density $n$ as a function of chemical potential $\mu$, temperature $T$, and further system parameters $\left\{g_{i}\right\}$ characterizing, for instance, the interactions between particles. For ultracold atoms with short-range attraction, the only additional parameter is the $s$-wave scattering length $a$. This universality allows one to describe different atomic species by the same EOS $n(\mu, T, a)$. The equilibrium EOS is also the basis for studying dynamics close to thermal equilibrium.

In this Letter, we report the experimental determination of the EOS of two-component fermions with attractive short-range interactions in the 2D BEC-BCS crossover regime. We tune the interaction strength using a Feshbach resonance to connect the well-known limits of a weakly attractive Fermi gas and a Bose gas of tightly bound dimers. We report the measurement of the finite temperature EOS in the intermediate, strongly correlated region and compare with theoretical predictions.

Our experimental setup consists of a populationbalanced mixture of $N \sim 100000{ }^{6} \mathrm{Li}$-atoms in the lowest two hyperfine states, which we denote by $|1\rangle$ and $|2\rangle$. The interactions between both species can be tuned by means of a magnetic Feshbach resonance [10,11]. The atoms are trapped in a highly anisotropic trapping potential, which is radially symmetric to a high degree in the $x y$ plane and provides a tight confinement along the $z$ direction with the aspect ratio of frequencies $\omega_{x}: \omega_{y}: \omega_{z} \approx 1: 1: 310$. A detailed description of the experiment is given in Ref. [7]. This strong anisotropy induces a quantum confinement of the many-body system, with a discrete excitation spectrum in the $z$ direction and an effective 2D continuum of states in the $x y$ plane. A 2D system is realized if the interacting system is in its ground state in the $z$ direction. Although the explicit form of this ground state is not known and depends on the $3 \mathrm{D}$ scattering length $a_{3 \mathrm{D}}$ [12], the ratio $N\left(\omega_{x} / \omega_{z}\right)^{2} \sim 1$ close to unity indicates that the first excited state in the $z$ direction will be partially populated. In this quasi-2D regime, interactions can still be described by an effective $2 \mathrm{D}$ scattering length $a_{2 \mathrm{D}}[6,13,14]$.

In order to parametrize the local density of the gas, we introduce the Fermi wave vector $k_{F}=(2 \pi n)^{1 / 2}$, where $n=$ $n_{1}+n_{2}$ is the planar density and $n_{\sigma}=n / 2$ is the density of atoms in state $|\sigma\rangle$. We further define the Fermi energy $\varepsilon_{F}=$ $\hbar^{2} k_{F}^{2} /(2 M)$ and Fermi temperature $T_{F}=\varepsilon_{F} / k_{B}$, where $\hbar$ is Planck's constant, $k_{B}$ Boltzmann's constant, and $M$ the atomic mass of ${ }^{6} \mathrm{Li}$. The $2 \mathrm{D}$ crossover [15-24] is parametrized by $\ln \left(k_{F} a_{2 \mathrm{D}}\right)$. In the BEC $\operatorname{limit} \ln \left(k_{F} a_{2 \mathrm{D}}\right) \ll-1$ the system can be described as a 2D Bose gas with effective coupling constant $\tilde{g} \approx-2 \pi / \ln \left(k_{F} a_{2 \mathrm{D}}\right)$. In the BCS limit $\ln \left(k_{F} a_{2 \mathrm{D}}\right) \gg 1$ the thermodynamic properties approach that of an ideal Fermi gas. In analogy to the 3D BEC-BCS crossover [25-28], the 2D gas undergoes a finite temperature phase transition to a superfluid state for all values of the parameter $\left(k_{F} a_{3 \mathrm{D}}\right)^{-1}$ [7]. The associated transition, however, is of Berezinskii-Kosterlitz-Thouless type and the superfluid phase exhibits quasi-long-range order [29-34].

For a 2D ultracold quantum gas with short-range attraction, a two-body bound state with binding energy 
$\varepsilon_{B}=\hbar^{2} /\left(M a_{2 \mathrm{D}}^{2}\right)>0$ exists for all values of the coupling strength. This has to be contrasted with the 3D case, where a bound state only exists on the Bose side of the resonance. In both cases, the chemical potential for a single fermion becomes negative in the Bose limit and approaches $\mu \approx-\varepsilon_{B} / 2$. The crossover point between the Bose and Fermi sides can be defined by the zero crossing of $\mu$ [35]. The chemical potential shifted by the bound-state energy, $\tilde{\mu}=\mu+\varepsilon_{B} / 2$, is positive at high phase-space densities. At zero temperature, $n>0$ is equivalent to $\tilde{\mu}>0$.

In the experimental realization of the quasi-2D gas in an anisotropic 3D trap, the interaction strength $a_{2 \mathrm{D}}$ depends on the typical momenta of scattering particles and thereby on the filling in the trap with axial frequency $\omega_{z}$. One can write $a_{2 \mathrm{D}}=a_{2 \mathrm{D}}^{(0)} e^{-\frac{1}{2} \Delta w\left(\tilde{\mu} / \hbar \omega_{z}\right)}$, where $a_{2 \mathrm{D}}^{(0)}$ is the scattering length in the dilute limit and $\Delta w$ is a positive function which reduces $a_{2 \mathrm{D}}$ at finite density $[6,13,36]$. The correction to $a_{2 \mathrm{D}}^{(0)}$ vanishes in the Bose limit where $\tilde{\mu} \rightarrow 0$, and becomes strongest in the Fermi limit where $\tilde{\mu} \simeq \varepsilon_{F}$. In our experiment we have $\hbar \omega_{z} / k_{B}=265 \mathrm{nK}$, which has to be compared with typical values $\tilde{\mu}_{0}=(40, \ldots, 200) \mathrm{nK}$ and $T=(60, \ldots, 25) \mathrm{nK}$ when going from the Bose to the Fermi limit. As most particles are in the center of the cloud, we approximate $a_{2 \mathrm{D}}$ and $\varepsilon_{B}$ by their central values using $\Delta w\left(\tilde{\mu}_{0} / \hbar \omega_{z}\right)$, giving $\Delta w \approx 0.2,0.9$, and 1.4 in the Bose, crossover, and Fermi regimes, respectively.

We extract the EOS of the homogeneous gas from the trapped system by using the local density approximation (LDA) which assigns a local chemical potential $\mu(\vec{r})=$ $\mu_{0}-V(\vec{r})$ to each point $\vec{r}$ in the trapping potential $V(\vec{r})$ [37]. Since $V(\vec{r})$ is known to a high precision, the homogeneous density $n(\mu, T)$ can be deduced from the measured local in situ density of the inhomogeneous system $n(\vec{r})=n\left(\mu_{0}-V(\vec{r}), T\right)$ once $\mu_{0}$ and $T$ have been determined [38]. The extraction of the homogeneous EOS from the trapped gas has been applied to both bosonic and fermionic systems and successfully compared with theoretical calculations [39-46].

Low-temperature EOS.-In order to determine the lowtemperature equation of state $n\left(\mu, T \rightarrow 0, a_{2 \mathrm{D}}\right)$ we extract $\tilde{\mu}_{0}$ from a Thomas-Fermi (TF) fit of the central region of the cloud. The TF model assumes locally $\varepsilon_{F}=c \tilde{\mu}$ for the central density region. This scaling is valid for large phase space densities (PSDs) $n \lambda_{T}^{2}$, where $\lambda_{T}=\left[2 \pi \hbar^{2} /\left(M k_{B} T\right)\right]^{1 / 2}$ is the thermal wavelength of atoms. We find that the prefactor $c$ only weakly depends on the temperature and fitting range at sufficiently low temperature and high densities, which confirms the validity of the linear relation $\varepsilon_{F} \propto \tilde{\mu}$ (see the Supplemental Material [36] for details). We fit $c$ in the intervals $I_{A}=[0.4,0.8] n_{\text {peak }}$ and $I_{B}=[0.5,1] n_{\text {peak }}$ for peak density $n_{\text {peak }}$, and define $\tilde{\mu}_{0}=\left(\tilde{\mu}_{0, A}+\tilde{\mu}_{0, B}\right) / 2$ as the average value of both outcomes.

In Fig. 1 we show the low-temperature EOS across the 2D BEC-BCS crossover in terms of $\tilde{\mu} / \varepsilon_{F}=1 / c$ vs $\ln \left(k_{F} a_{2 \mathrm{D}}\right)$, where $k_{F}$ corresponds to the peak density. The corresponding temperatures $(60-25 \mathrm{nK}$ from left to

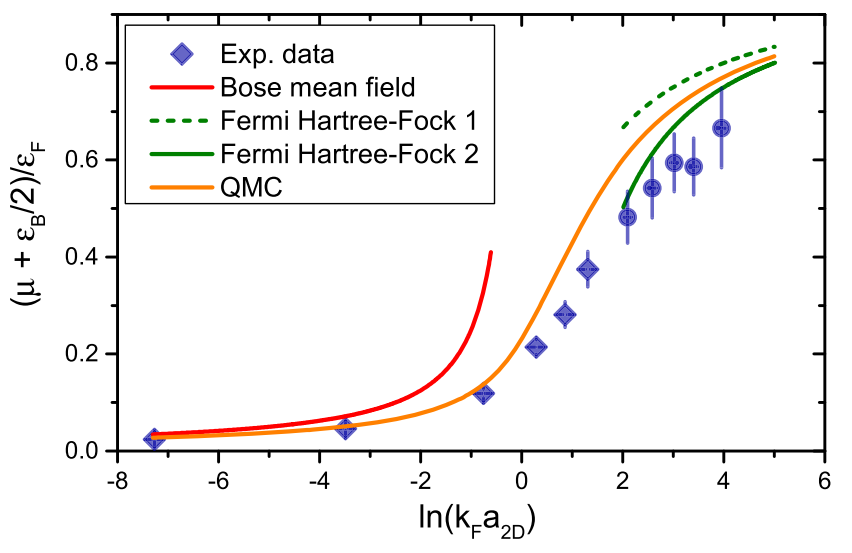

FIG. 1. Low-temperature EOS across the 2D BEC-BCS crossover. The experimental results are obtained from measurements of the quasi-2D gas at the lowest attainable temperatures, which corresponds to $T / T_{F} \approx 0.05$ and 0.1 on the Bose and Fermi sides. The data points shown as diamonds (circles) correspond to measurements in the superfluid (normal) phase. The solid red line on the Bose side corresponds to the mean field formula $\tilde{\mu} / \varepsilon_{F}=-\eta^{-1} / 4$ with $\eta=\ln \left(k_{F} a_{2 \mathrm{D}}\right)$, whereas the dashed and solid green lines on the Fermi side display the non-self-consistent and self-consistent Hartree-Fock predictions $1 /\left(1+\eta^{-1}\right)$ and $1-\eta^{-1}$ for weakly attractive fermions. The orange line is the prediction for the ground state EOS from recent QMC calculations [48].

right) are very low compared to $T_{F}(1500-300 \mathrm{nK})$. For the plot we averaged the TF slope $c$ over 30 images at the lowest temperatures for each value of the interaction strength. We find $c$ to be weakly dependent on temperature as we increase $T / T_{F}$ by (40-100)\%, which is a necessary condition for the applicability of the linear fit of the central region. The statistical error of $\tilde{\mu} / \varepsilon_{F}$ is $10 \%$ within the whole crossover. We estimate the error due to systematic uncertainties resulting from the absorption imaging, atoms in noncentral pancakes [47], magnification, and the determination of the binding energy to be $15 \%$ and $13 \%$ on the Bose and Fermi sides, respectively [7,36].

Our measured low-temperature equation of state connects both perturbative limits of the crossover. In the Bose limit we compare our results with predictions for bosonic dimers of mass $2 M$, dimer density $n_{d}=n / 2$, chemical potential $\mu_{d}=2 \tilde{\mu}$, and thermal wavelength $\lambda_{d}=\lambda_{T} / \sqrt{2}$. The interactions between dimers can be modeled by an effective 2D coupling strength $\tilde{g}=\sqrt{16 \pi}\left(0.6 a_{3 \mathrm{D}} / \ell_{z}\right)$ with $\ell_{z}=\sqrt{\hbar /\left(M \omega_{z}\right)}=0.551 \mu \mathrm{m}$ the oscillator length of axial confinement [49]. We find $\tilde{\mu} / \varepsilon_{F}=0.024(2), 0.046(4)$, 0.12 (1) for the data points with $\ln \left(k_{F} a_{2 \mathrm{D}}\right) \leq-0.71$ corresponding to effective boson coupling strengths $\tilde{g}=0.60$, $1.07,2.75$, respectively. This is in excellent agreement with the perturbative Bose gas formula $\tilde{\mu} / \varepsilon_{F}=\tilde{g} /(8 \pi)=0.024$, $0.043,0.11$. Furthermore, we verify $\tilde{g} \approx-2 \pi / \ln \left(k_{F} a_{2 \mathrm{D}}\right)$ for very small $\tilde{g}$, which is a result of $a_{2 \mathrm{D}} \simeq a_{2 \mathrm{D}}^{(0)}$ on the Bose side, where the filling correction $\Delta w$ is small.

Far on the Fermi side, our low-temperature data are consistently below the Hartree-Fock (HF) prediction 
$\tilde{\mu} / \varepsilon_{F} \simeq \mu / \varepsilon_{F}=1-\left[\ln \left(k_{F} a_{2 \mathrm{D}}\right)\right]^{-1}$. However, the additional error due to systematic uncertainties [36] is of the same size as the statistical one displayed in Fig. 1, such that we find consistency with HF theory within the errors of our measurements. Note that an extension of the BCS meanfield theory toward the crossover, which works reasonably well in $3 \mathrm{D}[50,51]$, would give $\tilde{\mu} / \varepsilon_{F}=1$ for all interaction strengths in the $2 \mathrm{D}$ case and thus clearly misses the crossover physics [52]. The ground-state equation of state has been investigated theoretically in Refs. [48,53-55]. In Fig. 1 we compare our measurements to recent QMC simulations of the ground state $[48,56]$. For a comparison of different theoretical predictions of $\tilde{\mu} / \varepsilon_{F}$ at zero temperature see, for instance, Ref. [55]. Our data at small but finite temperature lie consistently below the zero-temperature prediction.

Finite-temperature EOS.-While the temperature is constant within each atom cloud, it varies for every individual realization of the gas ("shot"). In our analysis we therefore determine $T$ and $\mu_{0}$ from each density profile and construct the dimensionless PSD $f_{i}(x, y)=f\left(\beta \tilde{\mu}, \beta \varepsilon_{B}\right)=n \lambda_{T}^{2}$ and normalized density $h_{i}(x, y)=h\left(\beta \mu, \beta \varepsilon_{B}\right)=n / n_{0}$ for every shot $i$. Here, $n_{0}(\mu, T)=2 \lambda_{T}^{-2} \ln \left(1+e^{\beta \mu}\right)$ is the EOS of an ideal Fermi gas. Finally, we average $f_{i}$ and $h_{i}$ over 30-150 shots to obtain the EOS with very small statistical error, even though the thermodynamic parameters vary from shot to shot. The values of $T$ and $\mu_{0}$ can be found by different methods: whereas the TF fit determines $\mu_{0}$ from the dense central region of the cloud, fitting a reference EOS to the outer low-density regions gives both $T$ and $\mu_{0}$.

We first summarize the reference EOSs used in this work. In the perturbative Bose limit of small $\tilde{g}$, the outer wings are described by the HF formula $n_{d} \lambda_{d}^{2}=$ $-\ln \left(1-e^{\beta \mu_{d}-(\tilde{g} / \pi) n_{d} \lambda_{d}^{2}}\right)$. The Boltzmann limit for a gas of dimers or atoms, respectively, reads $n_{d}=\lambda_{d}^{-2} e^{\beta \mu_{d}}=$ $2 \lambda_{T}^{-2} e^{2 \beta \tilde{\mu}}$ and $n_{\sigma}=\lambda_{T}^{-2} e^{\beta \mu}$. The latter two formulas are elegantly connected by the second order virial expansion $n_{\sigma} \lambda_{T}^{2}=\ln \left(1+e^{\beta \mu}\right)+2 b_{2} e^{2 \beta \mu}[35,36,57]$. In the weakly interacting Fermi limit $b_{2} \rightarrow 0$, and $n_{\sigma} \lambda_{T}^{2}$ approaches the EOS of an ideal Fermi gas. On the Bose side, instead, $\varepsilon_{B}$ becomes large and the fermion fugacity $e^{\beta \mu}=e^{\beta\left(\tilde{\mu}-\varepsilon_{B} / 2\right)}$ is extremely small, suppressing the first term of the EOS. However, $b_{2}=e^{\beta \varepsilon_{B}}$ up to exponentially small corrections and we recover the bosonic Boltzmann EOS $n_{\sigma}=$ $2 \lambda_{T}^{-2} e^{\beta\left(2 \mu+\varepsilon_{B}\right)}=\lambda_{d}^{-2} e^{\beta \mu_{d}}$. Hence, the second order virial expansion has the correct limiting behavior and provides a well-defined reference EOS throughout the crossover.

We apply the HF formula for the perturbative Bose gas to determine $T$ and $\mu_{0}$ only for $B=692 \mathrm{G}$ where $\tilde{g}=0.60$. For the remaining magnetic fields, $B[\mathrm{G}]=732-922$, the central chemical potential $\tilde{\mu}_{0}$ is determined from the TF fit of the central region. The temperature is estimated by $T=\left(T_{V}+T_{B}\right) / 2$, where $T_{V}$ and $T_{B}$ are obtained from second order virial and Boltzmann fits to the outer region, respectively. This choice is motivated by the observation that $T_{V}$ and $T_{B}$ give upper and lower bounds on the true

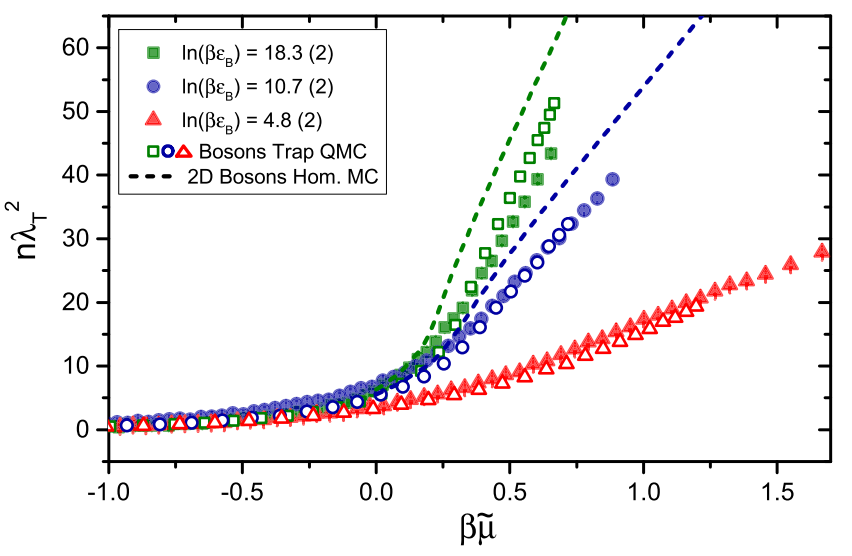

FIG. 2. Phase space density on the Bose side of the crossover. The experimental data points are shown as filled shapes. We compare to bosonic theory with effective coupling strengths $\tilde{g}=0.60,1.07,2.75$. Open shapes represent the EOS extracted from the QMC simulation of the quasi-2D Bose gas trapped in an external potential with similar parameters as employed in experiment. The dashed curves show the classical MC prediction for the weakly coupled homogeneous 2D Bose gas from Ref. [58] extrapolated to large values of $\tilde{g}$. For moderate densities we find good agreement between all three approaches. For large densities, however, experiment and trapped QMC calculations deviate from the classical homogeneous result which scales like the mean field prediction $n_{d} \lambda_{d}^{2}=(2 \pi / \tilde{g}) \beta \mu_{d}+\ln \left[(2 \tilde{g} / \pi) n_{d} \lambda_{d}^{2}-2 \beta \mu_{d}\right]$. This may be due to quantum effects appearing at large $\tilde{g}$, as well as the axial confinement.

temperature for the interaction strengths considered here. We quantitatively compare different methods to obtain $T$ and $\mu_{0}$ in the Supplemental Material [36]. In particular, we show that $\mu_{0}$ obtained from the virial and Boltzmann fits agrees well with the one from the TF fit, which also supports the validity of the TF assumption.

Bose EOS.-Figure 2 shows the EOS on the Bose side of the crossover. Because of the exponentially large binding energy in the Bose limit, the logarithmic dependence of the EOS on $\beta \varepsilon_{B}$ in $n \lambda_{T}^{2}=f\left(\beta \tilde{\mu}, \beta \varepsilon_{B}\right)$ can be replaced by the $\tilde{g}$ dependence in $n \lambda_{T}^{2}=F(\beta \tilde{\mu}, \tilde{g})$, where $F(x, \tilde{g})$ can directly be compared with bosonic theory for coupling $\tilde{g}$. The experimental data correspond to $\tilde{g}=0.60,1.07,2.75$. The plotted curves represent the average $F(x, \tilde{g})$ from approximately 30 individual shots for each interaction strength.

We compare our results on the Bose side to classical Monte Carlo (MC) and quantum Monte Carlo (QMC) simulations of bosons in order to understand whether the fermionic system can be described purely in terms of bosons. The classical MC computations are valid for the weakly coupled, homogeneous 2D Bose gas in the fluctuating regime $[58,59]$. In our case $\tilde{g}$ is large, and quantum effects are expected to modify the result. Even so, the critical temperature for $\tilde{g}<3$ is well described by extrapolating MC calculations to large $\tilde{g}[34,60,61]$. We also analyze density profiles obtained from QMC simulations of a trapped Bose gas with similar trapping parameters as in the experiment; see Refs. $[34,62,63]$ for details. We extract 

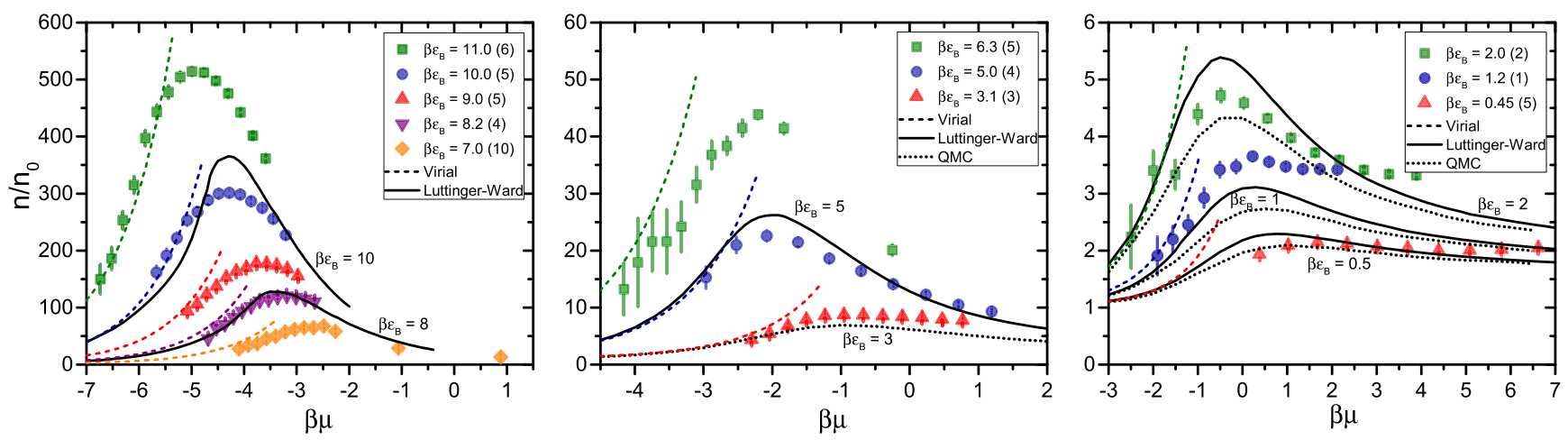

FIG. 3. EOS in the crossover regime shown as the density normalized by the ideal Fermi gas $n_{0}(\mu, T)=2 \lambda_{T}^{-2} \ln \left(1+e^{\beta \mu}\right)$. The experimental data points (filled shapes) are compared with the second order virial expansion at low values of $\beta \mu$ (coloured dashed lines). The displayed errors are purely statistical, with systematic uncertainties estimated at 13\%-15\% and given for each data set in Table IV in the Supplemental Material [36]. We compare our results with theoretical predictions in the 2D BEC-BCS crossover from Luttinger-Ward theory ([23], solid black lines) and fermionic QMC simulations ([24], dotted black lines), with the corresponding values of $\beta \varepsilon_{B}$ labeling each curve. Note that the vertical scale differs by a factor of 10 in each panel.

the temperature and chemical potential of the QMC density profiles from the low-density Boltzmann regime and apply the LDA to obtain the $\operatorname{EOS} F(\beta \tilde{\mu}, \tilde{g})$.

We find good agreement of our measurements with the EOS extracted from the QMC profiles. The deviations between both EOSs are within the systematic errors in the determination of $n, T$ and $\mu_{0}$ and we conclude that the fermionic system with $\tilde{g} \leq 2.75$ is well described by a strongly coupled quantum gas of dimers. Both experiment and QMC are, however, well below the classical MC predictions for large $\beta \tilde{\mu}$ and mean field theory. There are two effects which could explain this behavior: On the one hand, quantum fluctuations become important for large $\tilde{g}$ and high densities. On the other hand, both experiment and QMC simulations are performed in a quasi-2D setting with nonzero extent in the $z$ direction.

Crossover EOS.-Figure 3 shows the EOS in the strongly correlated crossover regime between the Bose and Fermi limits. The EOS $h(x, y)=n / n_{0}$ is sampled over approximately 150 shots for each of the magnetic fields $B[\mathrm{G}]=812,832,852,892$. We compare our results with theoretical predictions for the homogeneous 2D BEC-BCS crossover from Luttinger-Ward (LW) theory [23] and fermionic QMC simulations [24]. Our comparison covers a substantial renormalization by 2 orders of magnitude in the density $n / n_{0}$. It reveals a maximum in $n / n_{0}$ characteristic of the density driven crossover in 2D [23]. We find that the maximum of height $2 e^{\beta \varepsilon_{B} / 2}$ is reached at $\beta \mu \simeq-\beta \varepsilon_{B} / 2+$ $\ln (2)$ for large $\beta \varepsilon_{B}$. The origin of this scaling can be understood from the virial expansion in the Bose limit: $n_{\sigma} \lambda_{T}^{2} \approx 2 \exp (2 \beta \tilde{\mu})=2$ at $\beta \tilde{\mu}=0$, which implies $n / n_{0} \approx$ $2 / \ln \left(1+e^{-\beta \varepsilon_{B} / 2}\right) \approx 2 e^{\beta \varepsilon_{B} / 2}$ at $\mu=-\varepsilon_{B} / 2$; see the Supplemental Material [36] for details. The difference between the LW and QMC EOSs lies within our systematic errors from the $T$ and $\mu_{0}$ determination and thus cannot be resolved with the present analysis. In the Fermi limit where $\tilde{\mu}_{0} / \hbar \omega_{z} \lesssim 0.75$ is largest, we observe that the filling correction $\Delta w$ shifts the EOS slightly upward. This effect is minimized for small particle numbers. In a recent work by
Fenech et al. [46] the EOS in the normal phase for $\beta \varepsilon_{B}<0.5$ has been determined using ${ }^{6} \mathrm{Li}$ atoms in the $2 \mathrm{D}$ regime.

In this work we have measured the EOS of ultracold fermions in the BEC-BCS crossover in a strongly anisotropic confinement. Our results connect the perturbative Bose gas, the strongly interacting Bose gas, the strongly interacting fermionic superfluid in the crossover regime, and the perturbative Fermi liquid as we tune the effective 2D scattering length $a_{2 \mathrm{D}}$ using a Feshbach resonance. Our EOS data covers both the low and intermediate temperature thermodynamics of the system. We compare with bosonic and fermionic quantum many-body theory and find a remarkably strong renormalization of the density $n / n_{0}$. These results provide a basis for phenomenological computations such as hydrodynamic models of the cloud.

We gratefully acknowledge inspiring discussions with M. Bauer, M. Holzmann, T. Lompe, and J. M. Pawlowski, and thank J. Drut and H. Shi for sharing QMC data. This work has been supported by the ERC starting Grant No. 279697, the ERC advanced Grant No. 290623, the Helmholtz Alliance HA216/EMMI, and the Heidelberg Center for Quantum Dynamics.

*To whom all correspondence should be addressed. I.Boettcher@thphys.uni-heidelberg.de

[1] I. Bloch, J. Dalibard, and W. Zwerger, Rev. Mod. Phys. 80, 885 (2008).

[2] K. Martiyanov, V. Makhalov, and A. Turlapov, Phys. Rev. Lett. 105, 030404 (2010).

[3] P. Dyke, E. D. Kuhnle, S. Whitlock, H. Hu, M. Mark, S. Hoinka, M. Lingham, P. Hannaford, and C. J. Vale, Phys. Rev. Lett. 106, 105304 (2011).

[4] M. Feld, B. Fröhlich, E. Vogt, M. Koschorreck, and M. Köhl, Nature (London) 480, 75 (2011).

[5] A. T. Sommer, L. W. Cheuk, M. J. H. Ku, W. S. Bakr, and M. W. Zwierlein, Phys. Rev. Lett. 108, 045302 (2012). 
[6] V. Makhalov, K. Martiyanov, and A. Turlapov, Phys. Rev. Lett. 112, 045301 (2014).

[7] M. G. Ries, A. N. Wenz, G. Zürn, L. Bayha, I. Boettcher, D. Kedar, P. A. Murthy, M. Neidig, T. Lompe, and S. Jochim, Phys. Rev. Lett. 114, 230401 (2015).

[8] V. M. Loktev, R. M. Quick, and S. G. Sharapov, Phys. Rep. 349, 1 (2001).

[9] P. A. Lee, N. Nagaosa, and X.-G. Wen, Rev. Mod. Phys. 78, 17 (2006).

[10] W. Ketterle and M. W. Zwierlein, in Proceedings of the International School of Physics "Enrico Fermi," Vol. CLXIV (IOS Press, Amsterdam, 2008).

[11] G. Zürn, T. Lompe, A. N. Wenz, S. Jochim, P. S. Julienne, and J. M. Hutson, Phys. Rev. Lett. 110, 135301 (2013).

[12] P. Dyke, K. Fenech, T. Peppler, M. G. Lingham, S. Hoinka, W. Zhang, S.-G. Peng, B. Mulkerin, H. Hu, X.-J. Liu, and C. J. Vale, Phys. Rev. A 93, 011603(R) (2016).

[13] D. S. Petrov and G. V. Shlyapnikov, Phys. Rev. A 64, 012706 (2001).

[14] J. Levinsen and M. M. Parish, Annu. Rev. Cold Atoms and Molecules 3, 1 (2015).

[15] P. Bloom, Phys. Rev. B 12, 125 (1975).

[16] K. Miyake, Prog. Theor. Phys. 69, 1794 (1983).

[17] M. Randeria, J.-M. Duan, and L.-Y. Shieh, Phys. Rev. Lett. 62, 981 (1989).

[18] S. Schmitt-Rink, C. M. Varma, and A. E. Ruckenstein, Phys. Rev. Lett. 63, 445 (1989).

[19] J. R. Engelbrecht and M. Randeria, Phys. Rev. Lett. 65, 1032 (1990).

[20] M. Drechsler and W. Zwerger, Ann. Phys. (Berlin) 504, 15 (1992).

[21] D. S. Petrov, M. A. Baranov, and G. V. Shlyapnikov, Phys. Rev. A 67, 031601 (2003).

[22] M. Iskin and C. A. R. Sa de Melo, Phys. Rev. Lett. 103, 165301 (2009).

[23] M. Bauer, M. M. Parish, and T. Enss, Phys. Rev. Lett. 112, 135302 (2014).

[24] E. R. Anderson and J. E. Drut, Phys. Rev. Lett. 115, 115301 (2015).

[25] C. Chin, M. Bartenstein, A. Altmeyer, S. Riedl, S. Jochim, J. H. Denschlag, and R. Grimm, Science 305, 1128 (2004).

[26] M. W. Zwierlein, J. R. Abo-Shaeer, A. Schirotzek, C. H. Schunck, and W. Ketterle, Nature (London) 435, 1047 (2005).

[27] M. W. Zwierlein, C. H. Schunck, A. Schirotzek, and W. Ketterle, Nature (London) 442, 54 (2006).

[28] The BCS-BEC Crossover and the Unitary Fermi Gas, edited by W. Zwerger (Springer, Berlin, 2012).

[29] V. L. Berezinskii, Sov. Phys. JETP 32, 493 (1971).

[30] V. L. Berezinskii, Sov. Phys. JETP 34, 610 (1972).

[31] J. M. Kosterlitz and D. J. Thouless, J. Phys. C 6, 1181 (1973).

[32] Z. Hadzibabic, P. Krüger, M. Cheneau, B. Battelier, and J. Dalibard, Nature (London) 441, 1118 (2006).

[33] P. A. Murthy, D. Kedar, T. Lompe, M. Neidig, M. G. Ries, A. N. Wenz, G. Zürn, and S. Jochim, Phys. Rev. A 90, 043611 (2014).

[34] P. A. Murthy, I. Boettcher, L. Bayha, M. Holzmann, D. Kedar, M. Neidig, M. G. Ries, A. N. Wenz, G. Zürn, and S. Jochim, Phys. Rev. Lett. 115, 010401 (2015).

[35] V. Ngampruetikorn, J. Levinsen, and M. M. Parish, Phys. Rev. Lett. 111, 265301 (2013).
[36] See Supplemental Material at http://link.aps.org/ supplemental/10.1103/PhysRevLett.116.045303 for details on extracting thermodynamic parameters, discussion of systematic errors, and further theoretical background.

[37] L. D. Landau and E. M. Lifshitz, Statistical Mechanics I (Pergamon Press, Oxford, 1977).

[38] T.-L. Ho and Q. Zhou, Nat. Phys. 6, 131 (2010).

[39] M. Horikoshi, S. Nakajima, M. Ueda, and T. Mukaiyama, Science 327, 442 (2010).

[40] S. Nascimbene, N. Navon, K. J. Jiang, F. Chevy, and C. Salomon, Nature (London) 463, 1057 (2010).

[41] N. Navon, S. Nascimbene, F. Chevy, and C. Salomon, Science 328, 729 (2010).

[42] C.-L. Hung, X. Zhang, N. Gemelke, and C. Chin, Nature (London) 470, 236 (2011).

[43] T. Yefsah, R. Desbuquois, L. Chomaz, K. J. Günter, and J. Dalibard, Phys. Rev. Lett. 107, 130401 (2011).

[44] M. J. H. Ku, A. T. Sommer, L. W. Cheuk, and M. W. Zwierlein, Science 335, 563 (2012).

[45] R. Desbuquois, T. Yefsah, L. Chomaz, C. Weitenberg, L. Corman, S. Nascimbène, and J. Dalibard, Phys. Rev. Lett. 113, 020404 (2014).

[46] K. Fenech, P. Dyke, T. Peppler, M. G. Lingham, S. Hoinka, H. Hu, and C. J. Vale, preceding Letter, Phys. Rev. Lett. 116, 045302 (2016).

[47] We estimate that the presence of atoms in noncentral pancakes, i.e., neighboring sites of the standing wave potential, results in an overestimate of the planar density by at most $10 \%$ [36].

[48] H. Shi, S. Chiesa, and S. Zhang, Phys. Rev. A 92, 033603 (2015).

[49] D. S. Petrov, C. Salomon, and G. V. Shlyapnikov, Phys. Rev. Lett. 93, 090404 (2004).

[50] D. M. Eagles, Phys. Rev. 186, 456 (1969).

[51] A. Leggett, in Modern Trends in the Theory of Condensed Matter, edited by A. Pekalski and R. Przystawa (SpringerVerlag, Berlin, 1980), Vol. 115, p. 13.

[52] W. Ong, C. Cheng, I. Arakelyan, and J. E. Thomas, Phys. Rev. Lett. 114, 110403 (2015).

[53] G. Bertaina and S. Giorgini, Phys. Rev. Lett. 106, 110403 (2011).

[54] L. Salasnich and F. Toigo, Phys. Rev. A 91, 011604 (2015).

[55] L. He, H. Lü, G. Cao, H. Hu, and X.-J. Liu, Phys. Rev. A 92 , 023620 (2015).

[56] For the plot we use the piecewise defined interpolation function $f(x)$ with $x=\ln \left(2 e^{-\gamma} k_{F} a_{2 \mathrm{D}}\right)$ given in Ref. [48]. From $\mu=d \epsilon / d n$ we then find $\tilde{\mu} / \varepsilon_{F}=1+f(x)+\frac{1}{4} f^{\prime}(x)$.

[57] C. Chafin and T. Schäfer, Phys. Rev. A 88, 043636 (2013).

[58] N. Prokof'ev and B. Svistunov, Phys. Rev. A 66, 043608 (2002).

[59] N. Prokof'ev, O. Ruebenacker, and B. Svistunov, Phys. Rev. Lett. 87, 270402 (2001).

[60] L.-C. Ha, C.-L. Hung, X. Zhang, U. Eismann, S.-K. Tung, and C. Chin, Phys. Rev. Lett. 110, 145302 (2013).

[61] R. J. Fletcher, M. Robert-de Saint-Vincent, J. Man, N. Navon, R. P. Smith, K. G. H. Viebahn, and Z. Hadzibabic, Phys. Rev. Lett. 114, 255302 (2015).

[62] M. Holzmann and W. Krauth, Phys. Rev. Lett. 100, 190402 (2008).

[63] M. Holzmann, M. Chevallier, and W. Krauth, Phys. Rev. A 81, 043622 (2010). 